\title{
TÉMOIGNAGES
}

\section{Entretien avec Hugues Choplin et Arnaud Galisson}

Le centre de ressources en innovation pédagogique et technologie du groupe des Écoles des télécommunications vient de publier un rapport de recommandations pour concevoir et mettre en œuvre des dispositifs de formation ouverte et à distance. Distances et savoirs a souhaité demander à deux de ses auteurs de commenter certaines de leurs conclusions. Hugues Choplin et Arnaud Galisson appartiennent tous deux au département Innovations pédagogiques de Télécom Paris, Arnaud Galisson est le chef de ce département, Hugues Choplin y est le coordonnateur de la mission recherche.

Ils ont répondu aux questions de Monique Grandbastien, membre du conseil scientifique de Distances et savoirs durant l'été 2003.

Vous avez publié en décembre 2002 les conclusions du projet MIRéHD-EFAD ${ }^{1}$ sous la forme d'un rapport intitulé "Concevoir et mettre en ouvre des dispositifs de formation ouverte et à distance, rapport final de recommandations $»^{2}$. Pouvez-vous nous expliquer d'abord le contexte de ces travaux?

ARNAUD GALISSON - Le travail de MIRéHD-EFAD décrit ici se déroule dans un double contexte technologique. D'une part celui d'un travail de fond réalisé au sein du GET ${ }^{3}$ sur l'usage des technologies de l'information et de la communication pour l'éducation (initié en 1995 lors de l'appel d'offre sur les «autoroutes de l'information »), d'autre part, celui de la mise en place d'un réseau haut débit entre les écoles du GET qui devait supporter, entre autres, des échanges « lourds » dédiés à la formation des élèves.

Ce double contexte nous a amené à construire et mettre en œuvre cette expérimentation en quatre phases :

1. Projet « Multimédia interactif réseaux haut débit (MIRéHD), Expérimentation de formation à distance (EFAD) » (2000-2002).

2. H. Choplin (éd), A. Galisson, F. Méhat (Alcatel University), S. Morin (Télécom Paris), J.-S. Nouveau (Télécom Paris), D. Paquelin (Université de Bordeaux-3). Ce rapport est téléchargeable à l'adresse : http://www.ip.enst.fr/telechargement/mirehdefad.pdf

3. Groupe des Ecoles des télécommunications. 
- la définition de la notion d'un dispositif de FOAD 4 ,

- la conception et le développement de deux dispositifs de FOAD sur des thèmes scientifiques, selon le précédent modèle,

- la mise en œuvre expérimentale de ces dispositifs dans deux contextes distincts (un académique et un industriel),

- enfin, l'évaluation de ces mises en œuvre et la rédaction du rapport de recommandations.

Hugues ChOPLin - Elément complémentaire sur la notion de « dispositif de formation ouverte et à distance » : nous nous plaçons en effet davantage dans un cadre de formation ouverte (et à distance) que "simplement» dans un cadre de formation à distance. Par là, nous voulons nous démarquer aussi bien de la formation présentielle (basée sur une situation d'apprentissage privilégiée, de surcroît peu négociable : le cours traditionnel) que de la formation à distance (du moins celle basée également sur une situation d'apprentissage, peu susceptible d'aménagements : chez soi ou sur son poste de travail), lesquelles sont deux types de formation, fermées, qui ne réussissent probablement qu'avec un type de public très particulier... nous aurions envie de dire (dans le second cas au moins) avec une élite : des apprenants motivés et autonomes (ou métacompétents). De ce point de vue, formation présentielle et formation à distance : même combat! Ouvrir signifie donc pour nous: varier et articuler les situations d'apprentissage (sans les subordonner à une situation privilégiée) et rendre l'ensemble négociable, partiellement du moins (car il faut aussi probablement du non négociable). C'est bien cette négociation qu'implique pour certains l'idée de dispositif.

Quels ont été pour vous les principaux enseignements de ce projet? En particulier qu'avez-vous noté, formalisé de nouveau par rapport à ce qui était déjà connu en matière de FOAD?

Hugues CHOPLIN - Pour nous, le principal enseignement du projet est lié à la force des transformations que "subit» un dispositif de FOAD dans sa mise en œuvre, transformations par rapport au projet de conception. Dans la première expérimentation, l'autonomie des élèves a fait qu'ils n'ont pas sollicité comme c'était prévu le tuteur... qui semble du coup s'être trouvé isolé et perturbé. C'était lui qui était à distance! Il s'est du coup retrouvé dans la situation d'ajouter des séances de présentiel pour légitimer semble-t-il son rôle. C'était un phénomène vraiment étonnant, de la part d'un enseignant, réputé pour ses qualités pédagogiques, que de voir les élèves réussir normalement, mais « sans » lui... Dans la seconde, la transformation a été plus radicale encore. Le dispositif prévu était doublement collaboratif: au niveau du projet pédagogique (collaboration entre apprenants) comme au niveau de l'accompagnement (cinq accompagnateurs: un tuteur technique, un tuteur local chez l'industriel, un animateur pédagogique, un expert et

4. Formation ouverte et à distance. 
un responsable de formation devaient travailler ensemble pour «gérer» cet accompagnement). Or en une semaine, le dispositif de FOAD a été remis en cause par les apprenants avant de se transformer en des cours du soir «transmissifs » donnés par le tuteur local. La raison principale de cette transformation, ou plutôt de cette déformation (car les deux dimensions collaboratives ont « disparu »), tient très probablement à l'écart entre les deux mondes académique et industriel, qui ont respectivement « accueilli » la conception et la mise en œuvre.

Quelle nouveauté ? On sait bien sûr que la logique de l'usage n'est pas celle de la conception. L'écart a été ici très fort et a tenu à des éléments qui ne nous semblent pas souvent, ou pas suffisamment, relevés dans les recherches sur la FOAD : éléments en particulier de type sociologique, liés aux deux mondes sociaux différents engagés dans la seconde expérimentation (nous reprenons la formule de Bernard Blandin, elle-même héritée de Boltanski et Thévenot). Et puis, dans la première expérimentation, il y a, du côté du tuteur, la force de la dimension affective liée à l'absence imprévue des apprenants.

Quelles sont les recommandations que vous faites?

Hugues CHOPLiN — Les recommandations proposées (au nombre de 13) sont ordonnées autour de trois idées :

- déterminer l'acceptabilité et les objectifs d'un dispositif de FOAD,

-l'organiser des points de vue pédagogique, social, institutionnel et technologique,

- et réguler sa mise en œuvre, toujours complexe.

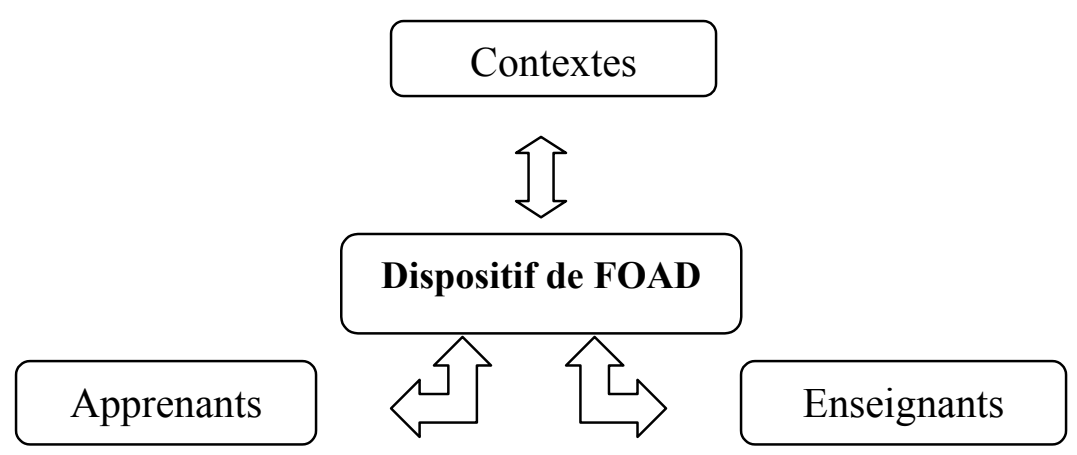

Figure 1. Le dispositif de FOAD et ses relations avec les trois pôles, " apprenants", " enseignants » et " contextes » 
Dans ces conditions, la recommandation générale tient au positionnement et au maintien du dispositif de FOAD dans une zone acceptable par les trois pôles. Pôle par pôle, les recommandations principales sont :

- pour le pôle apprenants : «profiter » de l'ouverture de la formation (en matière d'activités, d'espaces, de temps et de ressources humaines et technologiques) sans perdre les apprenants ; rompre leur isolement potentiel ; favoriser la possibilité qu'ils régulent eux-mêmes le dispositif de FOAD dans sa mise en œuvre ;

- pour le pôle enseignants : concevoir des ressources pédagogiques spécifiques à la FOAD s'appuyant sur les ressources traditionnelles ; favoriser l'appropriation du dispositif de FOAD par les enseignants-tuteurs via leur expérimentation et leur information/formation; organiser la collaboration et favoriser la régulation dans l'urgence (diagnostic et corrections éventuelles) de la mise en œuvre du dispositif de FOAD ;

- pour le pôle contextes : élaborer une stratégie ; relier les deux « mondes » de la conception et de la mise en œuvre (quand ils sont différents).

A ces recommandations, s'ajoutent celles, technologiques, de sélection et de mise en œuvre des outils d'échanges entre acteurs ainsi que de définition de la plateforme logicielle.

Pouvez-vous aussi revenir sur votre seconde recommandation: "Anticiper les coûts »?

Hugues ChOPLin - L'anticipation des coûts, le plus en amont possible, contribue à déterminer la faisabilité du dispositif, surtout si l'on anticipe (autant que possible) le coût temporel que peut prendre en particulier la négociation du dispositif, du moins la négociation de ses lignes générales ou structurantes (dominante pédagogique: tutorale, collaborative ou transmissive; type d'écranclé...) avec les différents acteurs concernés (apprenants compris). Cette anticipation est complexe parce qu'elle doit bien entendu s'imbriquer à une analyse pédagogique, de façon à montrer a priori les compromis à effectuer dans une perspective d'efficience. Et puis, cette anticipation ne doit pas omettre les coûts souvent masqués liés, par exemple, à l'administration de la plate-forme de $\mathrm{FOAD}$, à la sensibilisation des enseignants-formateurs ou encore à la mise à jour et à la maintenance du dispositif.

Comment situer ces recommandations par rapport à des méthodes générales de design pédagogique pour la FOAD?

ARNAUD GALISSON — Trois éléments spécifiques et originaux caractérisent ces recommandations :

- elles s'adressent à des acteurs opérationnels de la FOAD et ont été rédigées par des chercheurs en collaboration avec de tels acteurs opérationnels ; elles identifient en particulier en tant que tels les obstacles que posent la conception et la mise en œuvre de la FOAD ; 
- elles se basent sur une définition « à contre courant » de la formation à distance en la centrant non pas sur le document ou sur la plate-forme mais sur l'activité pédagogique de l'apprenant et ses espaces-temps. Par exemple, si l'on compare l'ingénierie sous-jacente proposée par «notre» département Innovation pédagogique à l'approche proposée par le LICEF, en particulier l'atelier (ADISA) et la méthodologie MISA, la conclusion serait que la méthodologie LICEF touche à l'ingénierie pédagogique des savoirs et des documents qui s'appuie sur l'hypothèse forte que la formation à distance est construite sur l'organisation didactique d'un seul document ou d'un ensemble de documents, alors que l'approche du département Innovation pédagogique porte sur l'ingénierie de la formation qui s'appuie sur l'hypothèse que la formation ouverte et à distance est construite sur l'organisation d'un ensemble souple et cohérent d'activités pédagogiques pour l'apprenant. Ces deux approches sont d'ailleurs plus complémentaires qu'en tension et le choix de l'une ou l'autre relève probablement d'une bonne analyse des trois pôles présentés dans le rapport ;

- troisième élément: elles s'appuient sur la spécification d'un processus d'ingénierie assez original dans le monde de la FOAD, qui consiste à réaliser une conception descendante: partir des objectifs de la formation, puis définir l'organisation de la formation, pour aboutir aux spécifications des documents ou aux choix de technologies. Ce processus veut s'inscrire dans une approche qui entend « concilier» industrialisation et conception ad hoc (plus proche en particulier des pratiques «artisanales» des enseignants). C'est dans cette perspective que nous défendons la nécessité, dans le processus de conception, d'une phase d'adaptation négociée du dispositif : le dispositif est déjà là, il a déjà été mis en œuvre une ou plusieurs fois, mais il s'agit quand même - autant que possible - de le renégocier avec les acteurs du contexte (prescripteur, apprenants, relais locaux) dans lequel il va être mis en œuvre... de façon à ne pas avoir, ensuite, de « mauvaises » surprises !

Vous aviez un regard de praticiens et aussi de chercheurs. Quelles sont les pistes de recherche qui vous semblent devoir être explorées prioritairement pour progresser?

Hugues CHOPLiN - Pour faire un peu de provocation : ce serait bien d'oublier un peu l'apprenant et l'enseignant... Plus sérieusement : nous faisons l'hypothèse qu'en complément des travaux, nécessaires, conduits sur les caractéristiques des apprenants (autonomie, motivation) et des enseignants (les compétences), il est nécessaire de développer des recherches, ou des « recherches-innovations » (selon la formule que nous utilisons avec G. Jacquinot pour se démarquer de la rechercheaction) sur des thèmes souvent identifiés comme déterminants en FOAD mais très rarement étudiés en tant que tels. Nous pensons aux thèmes des espaces-temps, des outils/objets (par exemple ceux mobilisés dans la conception pour configurer la formation : graphe de navigation, représentation spatio-temporelle de la FOAD) ou encore des collectifs (communautés d'apprentissage et/ou de pratique, réseaux d'entraide). Par exemple, on relève souvent les difficultés de gestion du temps et de l'espace qu'engendre pour les apprenants la FOAD (conflits entre espace-temps professionnel, espace-temps personnel et espace-temps de formation). Mais connaît- 
on la manière dont un apprenant se construit ses territoires, ses temps de formation ? La question vaut aussi sans doute pour l'enseignant-tuteur. De même, connaît-on la dynamique, par exemple collaborative, que suscitent les objets intermédiaires ou de transition (comme dit Bernadette Charlier) élaborés en cours de conception? Dans quelle mesure cette dynamique renvoie-t-elle à celle de groupes, considérés dans leurs dimensions sociocognitives mais aussi socio-affectives? Car les «fondements » affectifs des relations pédagogiques en général et des groupes en particulier nous semblent également constituer un thème aussi déterminant que peu examiné. La difficulté tient sans doute à ce que le traitement de ces différents thèmes exige de nouvelles méthodes de recherche, excédant le champ de la psychologie et des sciences de l'éducation pour convoquer en particulier les sciences de l'information et de la communication et la (psycho-)sociologie. Méthodes sans doute plus interdisciplinaires (pensez par exemple à la question de l'espace-temps !), peut-être aussi plus «cliniques ». C'est aussi cette nécessaire transformation de la recherche que veut marquer l'idée, ou l'exigence, de recherche-innovation.

Reste que de notre point de vue, la recherche-innovation pointe surtout la nécessité de former-accompagner les acteurs de terrain dans le déploiement de la FOAD. Car que ce soit en matière de références générales (dimension formation) ou au niveau de projet spécifique, contextualisé (dimension accompagnement), les besoins ou du moins les problèmes que rencontrent les acteurs nous semblent très importants ! C'est dans ce cadre que nous inscrivons notre action CAIFOD'5.

5. Cellule d'accompagnement à l'ingénierie de la Formation ouverte et à distance, animée par l'Université technologique de Compiègne, l'université d'Aix Marseille II et Télécom Paris. Cette cellule, mise en place par la direction de la technologie, s'inscrit au sein du « Réseau Appui Conseil et Expertises » au campus numérique. Cf. http://ip.enst.fr/caifod/ 